\title{
Émergence De Taxi-Motos Et Recomposition Spatio- Économique À Korhogo : Les Taxi-Villes Entre Stratégies D’adaptation Et Désespoir
}

\author{
Dindji Médé Roger \\ Géographe, Enseignant-chercheur, Assistant \\ Université Peleforo Gon Coulibaly de Korhogo / Labo VST (Côte d'Ivoire) \\ Diabagaté Abou \\ Géographe, Enseignant-chercheur, Maître Assistant, Université Félix \\ Houphouët-Boigny d'Abidjan / Labo LARES (Côte d'Ivoire) \\ Houenenou Kouadio Dénis \\ Géomaticien, Enseignant-chercheur, Assistant \\ Université Peleforo Gon Coulibaly de Korhogo / Labo LIST (Côte d'Ivoire) \\ Brou Émile Koffi \\ Géographe, Enseignant-chercheur, Professeur Titulaire \\ Université Alassane Ouattara de Bouaké / Labo VST (Côte d’Ivoire)
}

doi: 10.19044/esj.2016.v12n35p190 URL:http://dx.doi.org/10.19044/esj.2016.v12n35p190

\begin{abstract}
In this article we propose to assess the impact of the taxi motorcycle phenomenon on the functional organization of taxi-cities or taxicars in Korhogo; City in the North of the Ivory Coast. Taking advantage of the temporary banning of taxi-cities at the outbreak of the political-military crisis of 19 September 2002, these two-wheeled vehicles were able to find a place in public transport in Korhogo. Thus obliging the taxi-cities; previously only offering urban mobility, to find strategies of "reconquest". This reflection reveals that the number of taxi-cities has increased from 480 to 86 today. Their receipts have also increased from 11,500 to 6,500 CFA francs per day. In front of the "ground" entirely invested /occupied by taximotorbikes, taxi-cars had to create lines of services distributed on four stations in the city. The merit of this article is to focus on public transport in ivorian secondary cities. In order to do this, the method used is based on observations and land surveys conducted with actors involved in the management of urban transport in three Ivorian secondary cities: Korhogo, Bouaké and Ferkessédougou.
\end{abstract}


Keywords: Korhogo, emergence, motorcycle taxis, taxi-cities coping strategy

\section{Résumé}

Dans cet article nous nous proposons d’évaluer l'impact du phénomène taxi-moto sur l'organisation fonctionnelle des taxi-villes ou taxivoitures à Korhogo; ville du Nord de la Côte d'Ivoire. Profitant de l'interdiction temporaire des taxi-villes dès le déclenchement de la crise politico-militaire du 19 septembre 2002, ces engins à deux roues ont su se faire une place dans le transport collectif à Korhogo. Obligeant ainsi les taxivilles ; auparavant seule offre de mobilité urbaine, à trouver des stratégies de « reconquête ». Cette réflexion révèle que le nombre de taxi-villes est passé de 480 à 86 aujourd'hui. Leurs recettes sont également passées de 11500 à $6500 \mathrm{fcfa} /$ journée. Devant le «terrain » entièrement investi/occupé par les taxi-motos, les taxi-voitures ont dus créer des lignes de dessertes réparties sur quatre gares dans la ville. Le mérite de cet article, c’est de s’intéresser aux transports collectifs dans les villes secondaires ivoiriennes. Pour ce faire, la méthode employée s’appuie sur des observations et des enquêtes de terrains conduites auprès des acteurs impliqués dans la gestion des transports urbains dans trois villes secondaires ivoiriennes: Korhogo, Bouaké et Ferkessédougou.

Mots clefs: Korhogo, émergence, taxi-motos, taxi-villes, stratégie d'adaptation

\section{Introduction}

La crise militaro-politique de 2002 a favorisé l'émergence du phénomène moto taxi dans les transports socio collectifs, en Côte d'Ivoire. Il s'agit de motos organisées en service de transport en commun avec au moins un passager et très souvent de lourds bagages. Si Abidjan la capitale économique en est épargnée, plusieurs villes secondaires de la Côte d’Ivoire le vive. Parmi lesquelles, on note Korhogo. Par ailleurs, avec 226156 habitants (RGPH, 2014) Korhogo représente la principale ville de la région des savanes. En effet, cette région rassemble quatre départements occupants la partie septentrionale de la Côte d'Ivoire : (1) Korhogo, chef-lieu de région et capitale historique, commerciale et administrative de la zone; (2) Boundiali ; (3) Ferké et (4) Tengrela (TUO, 2013).

Cependant, il convient d'admettre que le taxi-moto apparait comme un mouvement à contre-courant de la tendance générale et locale en matière de transport urbain. En effet, au moment où dans les pays industrialisés on évolue des transports du type individuel vers le type commun dans un souci très écologique, à Korhogo les populations donnent de plus en plus leur 
préférence aux taxis motos qui sont d'une certaine manière un moyen de transport individualisé. Au niveau local, on peut se demander si une telle réalité cadre avec la vision du Gouvernement qui attend faire de la Côte d'Ivoire un "pays émergent ${ }^{8}$ " à l'horizon 2020. Depuis lors, la moto fait partie des moyens de déplacement des populations korhogolaises.

Ils sont visibles partout, postés à tous les carrefours et desservent tous les endroits de la ville. Aujourd'hui, les taxis motos occupent une place importante dans le transport collectif des personnes et des biens dans cette ville.

Cependant, leur forte présence n’a pas entrainé la disparition des taxis collectifs traditionnels qui pour leur survie ont réussi à s'adapter ; contraint d’accepter la concurrence. Mais, à la différence de Bouaké, la réalité de la concurrence est moins visible à Korhogo. En effet, dans la ville de Bouaké, la stratégie « concurrentielle » des taxis collectifs traditionnels ou taxis villes (taxi-voitures) est bien perceptible ; puisqu'ils parcourent la ville au même titre que les motos taxis à la conquête de clients. Par contre, à Korhogo, les taxis villes ont du s'organiser en quatre gares ou points de stationnement; attendant l'arrivée de clients. Tactique qui fait penser à première vue qu'ils ont perdu « bataille » devant les motos taxis. La nouvelle stratégie organisationnelle des taxis collectifs traditionnels ou taxis villes leur permet-t-elle de faire recette? Quelle était leur organisation fonctionnelle à Korhogo avant l'apparition des moto-taxis ? Quels sont les facteurs de l'émergence/consolidation du phénomène moto-taxi à Korhogo ?

Le mérite de cette réflexion, c’est de s’intéresser plutôt aux transports collectifs dans les villes secondaires ivoiriennes. De façon spécifique, elle se propose d'évaluer l'impact de l'émergence du phénomène moto-taxi sur l'activité des taxis collectifs traditionnels ou taxis villes ; lesquels assuraient exclusivement la desserte de l'espace urbain korhogolais.

\footnotetext{
${ }^{8}$ Conduite de grands projets devant conduire à une amélioration tangible du bien-être et à la qualité de la vie des ivoiriens.
} 


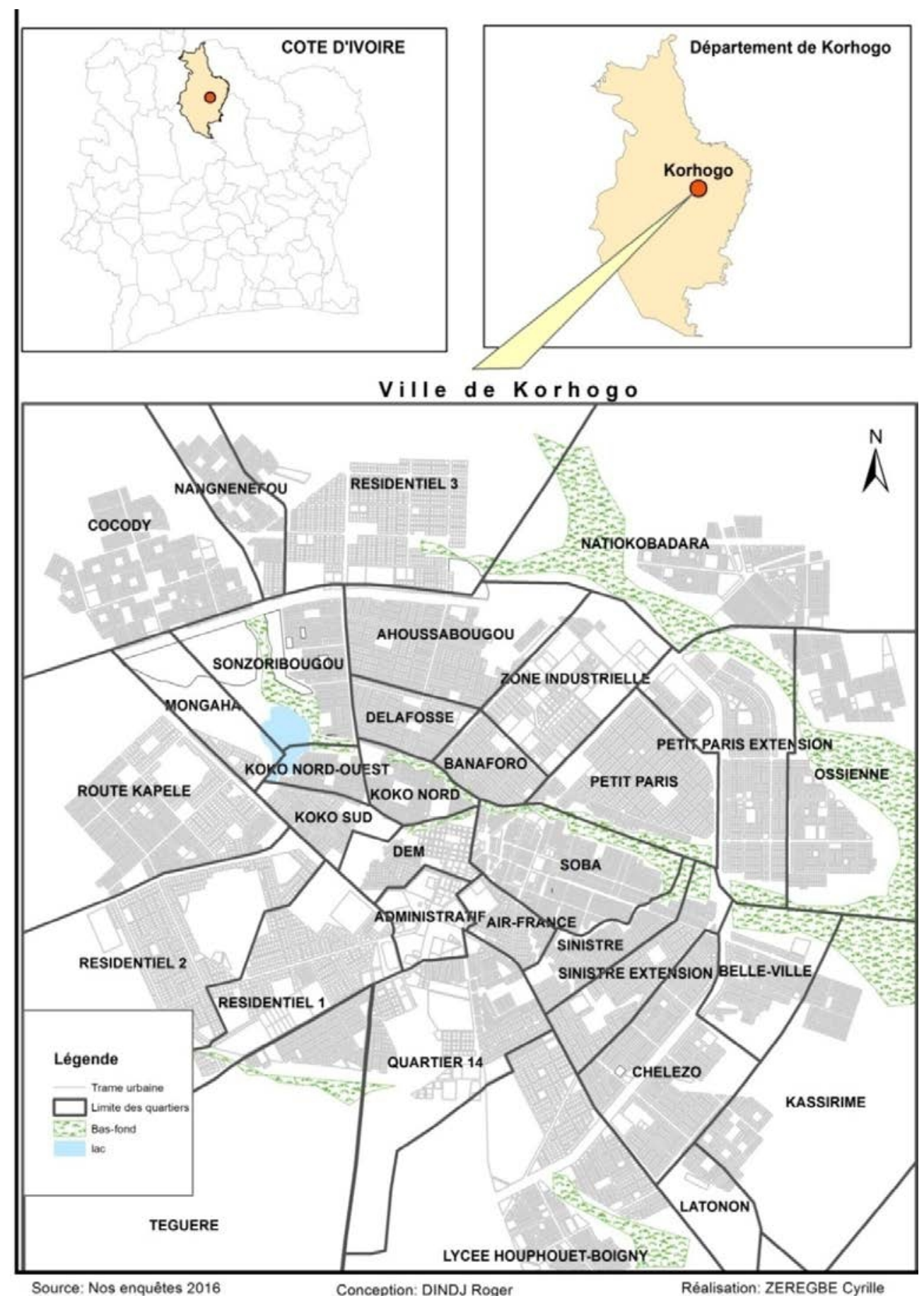

Figure 1 : Présentation de la ville de Korhogo et ses quartiers

La méthode employée s’appuie sur des observations et des enquêtes de terrains conduites auprès de tous les acteurs impliqués dans la gestion des 
transports urbains dans trois villes secondaires ivoiriennes: Korhogo, Bouaké et Ferkessédougou. Concrètement, des entretiens semi-directifs ont été réalisés avec les pouvoirs publics (municipalités, agents du ministère des transports) des trois localités suscitées, dans l’optique d’obtenir des informations et données en rapport avec la politique locale (la régulation) en rapport avec les transports collectifs. À cela, s’ajoute une enquête de terrain réalisée (de 2015 à 2016) sur la base d'un questionnaire administré aux principaux animateurs (responsables syndicaux, chefs de gares et chauffeurs de taxi-voitures et taxi-motos) des transports populaires de ces trois localités. Les leaders syndicaux nous ont renseignés principalement sur les points suivants : l'organisation et le fonctionnement de leur profession, états de leurs rapports avec les pouvoirs publics, les problèmes auxquels ils sont confrontés et surtout leurs perspectives. Avec les chauffeurs, les questionnaires ont été administrés dans le même esprit. Dans l'ensemble, nous avons eu recours à 100 enquêtés (pouvoirs publics, responsables syndicaux, chefs de gares, chauffeurs, etc.). L'analyse des données recueillies a permis d'organiser notre réflexion autour de deux points majeurs. D’abord, nous y faisons l'inventaire des facteurs de l'émergence du phénomène moto-taxi à Korhogo. Ensuite, vient l'analyse de la nouvelle organisation fonctionnelle des taxis villes ou taxi-voitures; pour une meilleur évaluation de l'impact des motos taxis sur l'activité de ce mode de transport.

\section{Les facteurs de l'émergence/consolidation du phénomène moto taxi à Korhogo}

L’apparition et la consolidation du phénomène moto taxi à Korhogo repose sur plusieurs facteurs. Pour une meilleure analyse, nous les avons regroupés en deux groupes : facteurs endogènes et facteurs exogènes.

\section{Les facteurs exogènes à l’émergence du phénomène moto taxi à Korhogo}

En côte d'Ivoire, le recours à la moto comme moyen de mobilité urbaine est un fait très récent. C'est d'ailleurs la raison pour laquelle, la majorité des villes ivoiriennes n’est pas encore affecté par cette réalité. Cependant, en Afrique, il ne s’agit pas d’un phénomène nouveau. En effet, depuis le début des années 90 plusieurs pays connaissent le taxi-moto. En Côte d'Ivoire, à l'instar de ces pays, l'avènement de ce phénomène trouve sa source principale dans des crises sociales (politique, militaire, économique, etc.). Pour ASSOGBA (2012) «les premières apparitions des taxi-motos à Lomé (capitale de la République du Togo) date des troubles socio politiques et c'est à partir de la grève illimitée du 12 novembre 1992 que ce nouveau mode de transport va s'emparer des togolais ». Au Cameroun également des villes comme Douala, Yaoundé, Bafousam, Dschang, Bamenda, etc. 
connaissent ce phénomène. Dans ce pays, la diffusion de la moto comme mode de transports collectif repose essentiellement sur la situation socio politique des années 1990 ponctuée par les différentes «...opérations de villes mortes » (PIERRE $\mathrm{K}$. et $\mathrm{Al}, 2007)$. Nous comprenons donc qu'en Afrique subsaharienne les grandes crises sociales (politique, militaire et économique) créées des opportunités de situations de tout genre ; à l’instar des motos taxis. À ce titre, la crise politico militaire déclenchée en Côte d'Ivoire dans la nuit du 19 septembre 2002 demeure la cause principale de l'apparition de ce phénomène dans ce pays en général et singulièrement à Korhogo. À ce facteur, s’ajoutent d'autres quoiqu'ils n'aient pas les mêmes effets. Ainsi, il faut compter avec l'insuffisance et/ou l'inexistence de services de transports publics dans de nombreuses villes africaines.

La plupart des villes africaines connaissent des problèmes dans le domaine du transport public. Ces problèmes y ont comme manifestation la fermeture des entreprises de transport intra urbain. Enfin, pour celles qui en disposaient. À ce propos, TUBLU (2010) rapporte que «depuis 1982, il n'existe plus des transports collectifs urbains à Lomé pas plus que dans les villes de l'intérieur qui n'en ont jamais eu d'ailleurs. Devant ce vide, des taxis artisanaux se sont implantées pour desservir le centre ville de Lomé et des points spécifiques de la ville ». Cette réalité n’est pas très loin de celle de la Côte d'Ivoire. Sauf que dans ce pays la Société des Transport Abidjanais (SOTRA) tient toujours la route. Cependant, depuis la décennie 1980, la qualité de son service a baissé en efficacité. En effet, la population abidjanaise évolue à un rythme très croissant et le potentiel en bus de cet opérateur est décroissant. Ce qui a favorisé l'émergence dans cette mégalopole de transports artisanaux (gbakas, wôrôs-wôrôs, pinasses, etc.) pour suppléer la demande en transport à satisfaire. De plus, la SOTRA ne couvre que la ville d'Abidjan et sa périphérie Nord-est (Bingerville). Ainsi, les populations des villes secondaires du pays ont recours aux taxi-villes ou taxis voitures; lesquels aujourd'hui livrent concurrence avec les motos; notamment à Korhogo. Dans ces villes africaines (motos taxis), le fonctionnement de la moto comme taxi présente quelques avantages qui ont facilité son adoption par les populations. Ces avantages se perçoivent à l'analyse de la flexibilité dans le parcours/trajet, le coût et l'accessibilité. Le taxi moto exprime/représente une nouvelle forme de mobilité à la demande. Il s’agit pour le client/passager de donner sa destination en fonction de quoi un prix lui est proposé par le chauffeur (TUBLU, 2010). Il faut reconnaître qu’à Korhogo le prix n'est pas toujours un avantage. En effet, le prix minimal pratiqué par les moto-taxis, c'est $200 \mathrm{fcfa} / \mathrm{client}$. Or, certains parcours intra urbain vont jusqu’à $500 \mathrm{fcfa} / \mathrm{client}$. Ce qui n’est pas le cas avec les taxi-villes ou taxi-voitures. Qu'importe la destination le tarif de 200 fcfa/client ne varie pas. La tarification ne varie que lorsqu'il est pris en 
« course ». Si tel est le cas le client paie au moins le tarif de tous les passagers (les quatre places). Nous admettons tout de même que la possibilité de négoce entre passager et chauffeur demeure un avantage. La flexibilité des parcours/trajets donne la possibilité au client/passager de se faire conduire jusqu'à sa porte même si son domicile n'est pas situé sur une grande artère. Enfin, le taxi moto facilite l'accès aux zones urbaines difficiles d'accès. En effet, ce mode de transport a l'avantage de se déplacer aisément sur les pistes et sentiers, de gravir les collines en toute saison et déposer les clients/passagers à domicile (PIERRE K. et Al, 2007). Dans un tel contexte, le taxi moto gagnerait facilement la concurrence sans aprioris contre le taxiville (ou taxi-voiture) ; tout en montrant leur utilité. Voici autant de facteurs qui ont favorisé l'émergence/consolidation du phénomène moto-taxi dans les agglomérations africaines en général et à Korhogo en particulier. Mais, il faut admettre que des facteurs locaux ont facilité cette réalité.

\section{Les facteurs endogènes de l'émergence du phénomène moto taxi à Korhogo}

\section{Korhogo : une ville de plus en plus attractive}

Korhogo est une ville en (re)construction, puisque sortant de crise. Mais, elle a gardé son attractivité d’alors. En effet, de 1965 à 1975, la ville de Korhogo connaissait l'une des croissances démographiques les plus fortes du pays, d'environ 7 \% l'an. Sur cette période, la moyenne des onze plus grandes villes de Côte d'Ivoire était de 5,7\%. De 20800 habitants en 1963 à 47500 en 1975 (TUO, 2013), la population urbaine est passée à près de 110 000 en 1988, 150000 en 1998 et à 286071 en $2014\left(\mathrm{RGPH}^{9}\right)$. Pendant cette période, la ville de Ferkessédougou, concurrente potentielle de la capitale régionale, n’a pas réduit l’attraction korhogolaise malgré l’implantation du rail et la création de grandes unités agro-industrielles : Ferkessédougou est restée une « ville de passage » et Korhogo une « ville de destination ». Il reste que les causes de l'urbanisation rapide de Korhogo sont : (a) les migrations de jeunes ruraux du département, attestées d'ailleurs par un relatif tassement de la croissance dans la «zone dense » et l'ouverture d'un front de colonisation des terres vers le Bou et le Bandama ; (b) un taux de natalité en ville (55\%00) plus élevé qu’en zone rurale (46\%00), tenant au taux élevé de femmes de moins de trente ans ; (c) la fonction commerciale, industrielle (sociétés COIC, EKDS, URECO-CI) et administrative de la ville qui, liée à son histoire, en fait un carrefour et un lieu d'attractions (TUO, 2013). Depuis 2012, une université (à caractère agro pastorale) a été ouverte dans cette ville. Chaque année, elle est pourvue en étudiants et en personnel (enseignant, technique et administratif). Du coup, la forte population de la

\footnotetext{
${ }^{9}$ Recensement Général de la Population et de l’Habitation.
} 
ville (y compris celle des villages et villes environnants) représente une clientèle dont la mobilité peut être satisfaite par les motos taxis. Par ailleurs, la baisse des prix des engins à deux roues à faciliter la consolidation du phénomène taxi-moto à Korhogo.

\section{La guerre comme porte d'entrée de marque de motos à prix plus accessible}

À partir du 19 septembre 2002, la ville était sous le contrôle d'une rébellion armée. Cette situation a favorisé la naissance de plusieurs trafics. Lesquels ont été favorisés par la souplesse dans la gestion des deux frontières Nord du pays : avec le Mali et le Burkina Faso. Ainsi, plusieurs marques (Saman, Dayun, Sanya, Samsung, Royal, Apsonic, etc.) jusque là méconnues ont eu accès au marché ivoirien ; notamment par le Nord. Concrètement, dans une ville comme Korhogo, l'offre en matière de motos s'accroît considérablement d'une part et ; y facilite l'acquisition des engins à deux roues d'autre part.

Ainsi, ces marques d'origine chinoise mènent la concurrence sur un " espace " précédemment acquis pour les marques françaises et japonaises (Peugeot, Suzuki, Yamaha, etc.). Selon nos enquêtés, la moto « super $100 »{ }^{10}$ qui coûtaient $1500000 \mathrm{fcfa}$, aujourd'hui on peut l'obtenir à Korhogo à 250 000-300 $000 \mathrm{fcfa}$. À ce facteur local, s'ajoute «le laisser-faire » des gestionnaires de villes.

\section{Le "laisser-faire " des gestionnaires urbains vis-à-vis du phénomène moto taxi}

Les chauffeurs de motos taxis bénéficient de la « collaboration » des " gestionnaires de villes ». Cette réalité s'explique en plusieurs points. Après la fin de la belligérance entre les différentes forces (étatiques et rebelles) de la crise ivoirienne, il fallait trouver une occupation aux ex-combattants. Parmi ces derniers, on dénombre plusieurs jeunes. Dès lors, le taxi-moto est devenu un outil excellent d'insertion d'une jeunesse passive et encombrante pour certains. Ainsi, dans plusieurs villes dont Korhogo des motos et tricycles ont été offerts aux ex-combattants. Un tel contexte ne favorise pas l'établissement d'une véritable politique de régulation de ce mode de transport. Il en ressort que les taxi-motos ne sont pas soumis aux mêmes normes de contrôles que les taxi-villes de la part des forces de l'ordre. À la réalité, ils ne sont pas contrôlés. Il en résulte que le nombre des motos taxis croit au jour le jour. En fait, il ne faut pas grande chose pour faire un tel travail. La connaissance du code de la route et la possession du permis de conduire ne sont pas nécessaire. Dans les villes de Korhogo et Bouaké, plus

\footnotetext{
${ }^{10}$ Appellation locale des motos à vitesse des motos françaises et japonaises.
} 
de $80 \%$ des accidents de la circulation seraient causés par ce nouveau mode de transports urbains. Malgré cela, le taxi-moto est reconnu par les municipalités de ces deux localités.

Des propos de quelques chauffeurs de motos taxis : «dans le temps avant d'exercer cette activité, il faut disposer d'une 'autorisation de circuler. Elle est délivrée par la mairie de Korhogo après que le propriétaire de la moto s'y fasse identifier. Pour cela, on paie $11000 f c f a$. Cette autorisation est matérialisée par le "'macaron de couleur jaune" que le chauffeur colle sur sa moto». Par ailleurs, lors de l'identification, les informations prises en compte concernent « le reçu d'achat de la moto et l'identité du propriétaire de la moto ». Autrement dit, le permis de conduire et la présentation du/des casque(s), l'immatriculation de la moto ne sont pas pris en compte.

Cependant, tous les mois le propriétaire de la moto paie aux services transports de la mairie de Korhogo la somme de 2 500fcfa. Par contre, à Bouaké ils paient 3 000fcfa. De ce point de vue, les taxi-motos contribuent à renflouer un temps soit peu les caisses municipales. Cependant, depuis le 26 janvier 2015, une note émanant du deuxième adjoint au maire de la ville donne «les conditions pour la en circulation ou le renouvellent de taxi-moto et tricycle. Pour cela, il faut: un certificat de nationalité ou carte consulaire, un casier judiciaire, un registre de commerce, carte grise, la vignette, l'assurance, deux photos du propriétaire, peindre la moto en jaune, une demande à retirer à la mairie (coût imprimé : 1 000fcfa), l'autorisation de transport publique (10 000fcfa), le permis de conduire (catégorie A)». Cette mesure démontre que la municipalité s’efforce pour légaliser ce mode de transport. Cependant, cette note a été prise seulement en janvier 2015. Pourtant, le phénomène a court dans la ville depuis plus de dix ans. On peut se demander pourquoi une telle mesure maintenant ? C'est dire qu'elle est restée pendant longtemps flexible et tendre vis-à-vis des acteurs de ce type de transports. Et si elle décide de le réguler, elle ne peut devenir rigide rapidement. En clair, elle peut accepter le fonctionnement dans les mêmes conditions avant l'émission de cette mesure. Il en ressort de l'analyse cidessus que la flexibilité des autorités locales a favorisé l'intégration et la consolidation du phénomène dans la ville de Korhogo. Toutefois, il faut admettre que les engins à deux roues ne sont pas étrangers au quotidien des populations locales.

\section{Un contexte local favorable à la moto}

Le phénomène moto-taxi était méconnu dans la ville avant la guerre. Ce qui n'est pas le cas pour la moto. En fait, cet engin a toujours fait partie du quotidien des populations du grand Nord ivoirien. C'est d'ailleurs ce qui explique l'absence de ce phénomène dans la ville concurrente et voisine à 
Korhogo : Ferkessédougou. En effet, de nos enquêtes nous retenons que la municipalité de Ferkessédougou aurait bien aimé avoir quelques moto-taxis pour renflouer ses caisses. Mais, elle ne peut vivre une telle réalité, puisque dans cette localité « tout le monde (paysans, fonctionnaires, élèves, jeunes, vieux, etc.) possède une moto ». Ainsi, les populations de la région étant habituées, ce phénomène n’a pas eu du mal à être accepté. Par ailleurs, pour la mobilité des populations des villages de Korhogo, en plus des motos, les populations ont recours aux tricycles. Malgré, les risques permanents d'accidents, d'insécurité (agressions), le phénomène moto-taxi fait désormais parti des moyens de déplacement par excellence des populations. Situation qui affecte considérablement l'organisation et le fonctionnement des taxivilles ou taxi-voitures dans la ville de Korhogo.

\section{L'organisation fonctionnelle des taxi-villes face à la concurrence des taxi-motos à Korhogo}

Les taxi-villes entre « l'auto réglementation » et le « laisser-faire » des autorités locales

L'activité des taxi-villes est organisée par les chauffeurs eux-mêmes à travers le Syndicat Communal des Chauffeurs de Taxi-Villes de Korhogo (SCCTVK). Des propos de nos enquêtés, cette organisation a toujours existé. Cependant, son actuel bureau dirigeant date de 2011. Ce bureau a été mis en place à la suite d'une élection. À la réalité, le bureau est constitué par le président élu. Autrement dit, la présidence est l'unique poste électif. Cette élection s'est tenue à la chambre des métiers de Korhogo (juste après les élections présidentielles de 2010) (notre enquête, 2015). Les votants devaient disposer de la carte professionnelle ou du permis de conduire. Auparavant, ils ont tous été recensés dans les différentes gares de taxi-villes de Korhogo. Depuis lors, le bureau de cette association est constitué par ces entités : un président (SORO Senisongou), un vice-président (COULIBALY Tanna), un secrétaire général et son adjoint, les chefs de gares et leurs adjoints, un trésorier général et son adjoint, un commissaire aux comptes et son adjoint. Le bureau siège à la gare centrale (à la gare de petit Paris) près du marché de gros de Korhogo.

Concrètement, les missions assignées par les chauffeurs de taxi-villes à leur organisation sont les suivantes « la représentation des chauffeurs à l'extérieur, la coordination des actions d'entraide et de solidarité entre les chauffeurs, la conduite de plaidoyer auprès des autorités pour l'amélioration des conditions de vie et de travail des chauffeurs ; notamment devant la prolifération incontrôlée des moto-taxis dans la ville, la gestion des conflits/incompréhensions entre chauffeurs et propriétaires, l'organisation du travail dans les gares de la ville, etc. ». Pour l'atteinte de ses missions la caisse du SCCTVK est alimentée par deux sources : le droit 
de ligne ordinaire et la cotisation hebdomadaire (de 500fcfa) versée par tous les chauffeurs les jours de marchés.

En effet, avant l'intégration d'un véhicule dans une gare son propriétaire paie la somme de 10 000fcfa (comme frais ou droit de ligne). Mais, nous y reviendrons plutard.

Retenons que le bureau du Syndicat est constitué par les responsables des différentes gares et points de stationnements de la ville. C'est donc ce syndicat qui régule l'organisation et le fonctionnement du transport des taxivilles ou taxi-voitures à Korhogo. À ce titre, avant d'exercer dans ce secteur, il faut disposer d'un véhicule (peint en rouge : couleur des taxis collectifs de la ville de Korhogo). Ensuite, formuler la demande auprès de ce bureau. À cette demande, il faut adjoindre le droit de ligne (10 000fcfa) et la gare de rattachement de la voiture.

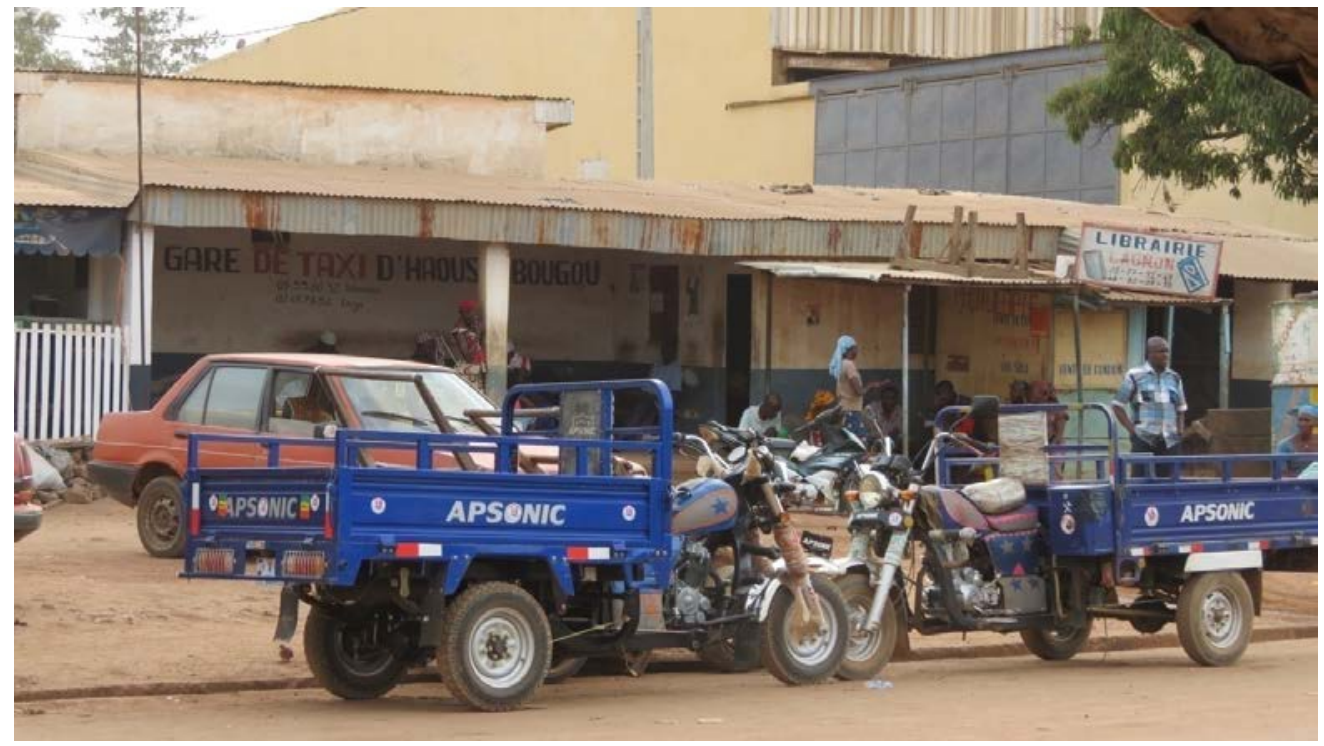

Cliché : DINDJI Roger, 2015

Figue 2 : un aperçu de la gare des taxi-voitures de Haoussabougou

Toutefois, si le propriétaire change de véhicule, il est obligé de payer à nouveau le droit de ligne. Par ailleurs, des propos des responsables syndicaux «lorsque le demandeur rempli toutes les formalités, il est autorisé à rouler ».

\section{L'intervention des gestionnaires de ville dans l'activité des taxi-villes}

Avant la crise (de 2002), le fonctionnement d'un taxi-ville à Korhogo exigeait l'enregistrement (du véhicule et du propriétaire) auprès de trois entités: la municipalité, le service des impôts d'Etat et le syndicat des chauffeurs. Auprès de la municipalité, tous les mois, les chauffeurs payaient un timbre (500fcfa). Ce timbre était collé sur la carte. À cela, s'ajoute la 
« carte de mairie » au prix de 3 000f/mois. Par contre, avec les impôts les chauffeurs prenaient les documents ci-après : la patente (135 000fcfa/an), la vignette 25000 ou 30 000f selon la puissance de la voiture. Par ailleurs, la voiture devait avoir la « carte de visite technique » (14 000 ou 15 000fcfa) et «l'assurance » (au prix de $18200 \mathrm{fcfa} / \mathrm{mois}$ pour les 08 chevaux et $15000 \mathrm{fcfa}$ pour les 05 et 07 chevaux). Le propriétaire devait également disposer d'un « registre de commerce ». Après l'identification par la municipalité et les impôts, les chauffeurs pouvaient s'adresser à leur syndicat. Aujourd'hui, les chauffeurs de taxi-villes ont un autre regard vis-àvis des autorités. Plusieurs facteurs expliquent cela. Ils se sentent délaisser et mépriser par ces derniers au détriment des taxi-motos et tricycles. Pour eux les «trois roues et deux roues ne seraient pas contrôlées par les forces de l'ordre quand ils sont en infraction. Ils exerceraient sans vignette, assurance et visite technique. Et malgré cela, ils ne sont pas inquiété »].

En conséquence, depuis l'avènement du phénomène moto-taxi à Korhogo les chauffeurs de taxi-villes ne paient ni patente (aux impôts) ni une quelconque redevance à la municipalité. En clair, depuis 2002, les taxi-villes ont rompu tout contact avec la municipalité et les services des impôts d'Etat. Leur corporation syndicale demeure donc le principal régulateur de leur activité.

\section{L'impact des taxi-motos sur l'organisation fonctionnelle des taxi- voitures à Korhogo}

Aujourd'hui, l'organisation fonctionnelle des taxi-villes ou taxivoitures repose inéluctablement sur quatre gares. Il s’agit de la gare du Centre Hospitalier Régional (CHR), de celle de Napié et de celles du centre ville (petit Paris et Haoussabougou).

La gare de petit Paris ne date pas d'aujourd'hui. Elle a toujours existé. Cependant, avant la guerre, c’était juste un «point de stationnement ${ }^{11}$ ». En fait, aujourd'hui les taxi-villes refusent de parcourir la ville pour la quête de clients. Pour ces derniers cela est peine perdue, puisque les taxi-motos ont déjà quadrillé la ville assiégeant tous les carrefours. Les espaces ou points qui servaient de repos temporaires aux chauffeurs sont devenus aujourd'hui des gares. C'est là qu'ils attendent les clients.

Par ailleurs, avant la guerre, cette gare était plus proche du marché. Elle fut délocalisée par les autorités rebelles. La gare de «petit Paris » dessert 17 lignes: (1) petit Paris, (2) Kassirimé, (3) le quartier 14, (4) Résidentiel, (5) Aushénais, (6) Natchio, (7) Belleville, (8) Promofolo, (9)

\footnotetext{
${ }^{11}$ Une tête ou point de stationnement désigne un endroit où des arrêts sont marqués pour débarqués des clients et en embarqués d'autres, In Décentralisation et développement local : cas de la commune de Cocody, thèse unique de doctorat de Géographie, Abidjan-IGT, 2014, $382 \mathrm{p}$.
} 
Zone, (10) Kokoh, (11) Ahoussabougou, (12) Sozorbougou, (13) Cocody, (14) Nagnerofo, (15) Nouveau quartier, (16) Mongaha, (17) Brofohakaha (dans le quartier Cocody).

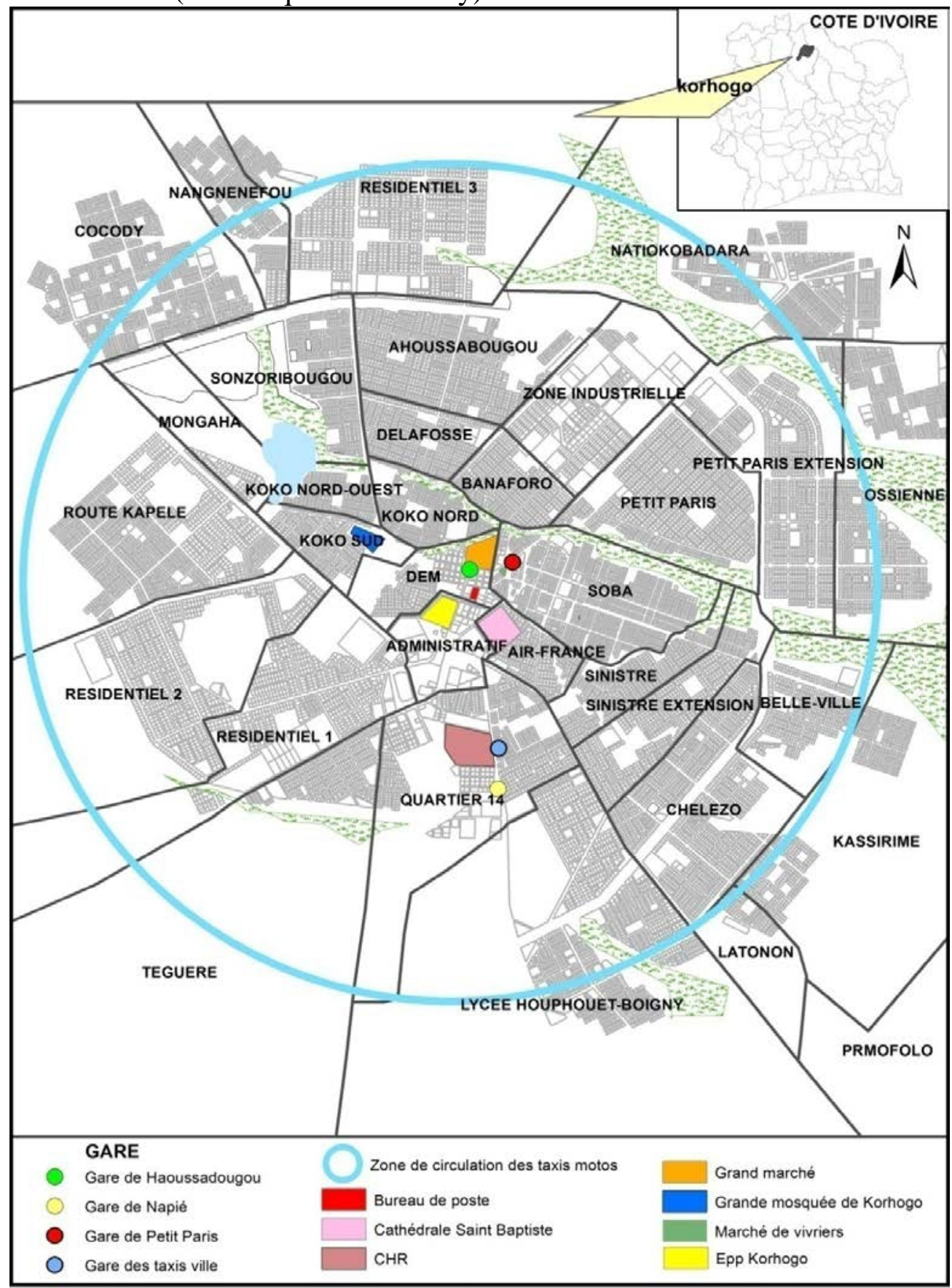

Source: Nos enauêtes $2016 \quad$ Concention: DINDJ Rocer Réalisation: ZEREGBE Cyrille Figure 3 : Cartographie de la nouvelle organisation fonctionnelle des taxi-voitures à Korhogo 
Concernant la gare de «Haoussabougou », elle est située du côté Sud du marché central de Korhogo. Ses taxi-villes desservent les lignes suivantes: (1) Sossoroubougou, (2) Cocody, (3) Delafosse, (4) Trois poteaux/nouveau quartier. Pour ce qui concerne la gare [CHR], elle doit son changement de statut à l'émergence des taxi-motos. En fait, avant 2002, cette gare n'existait pas. À ce jour, on y dénombre environ 20 véhicules. De ce site, tous les quartiers de la ville sont desservis. Les chauffeurs de cette gare ont la particularité de travailler jusqu’à des heures tardives pour assurer la mobilité de malades et de personnes qui leur porte assistance. La dernière des gares, c'est celle de Napié. Elle fut crée en 2003 par quelques chauffeurs en quête de réinsertion. En fait, après le déclenchement de la crise en 2002, la circulation fut interdite aux taxis collectifs par les autorités militaires qui contrôlaient la ville.

À la reprise, l'insertion n'était pas facile. D'où l'orientation par certains chauffeurs vers cette gare qui ne dessert que la campagne. Principalement, cette gare a deux lignes de dessertes : Napiéolodougou et Dikodougou. Il ressort de nos enquêtes qu'une trentaine de véhicules y sont en fonction.

Dans les gares à destinations urbaines, l'organisation du travail est le même. Les départs/arrivées, etc. se font sous la supervision d'un chef de gare. Les départs ne se font pas par ordre d'arrivée. En effet, une fois qu'un véhicule arrive en gare, il peut repartir aussitôt. Il revient à son chauffeur d'informer ses collègues et les clients de sa prochaine destination. Et il le signifie en le disant tout haut. La tarification appliquée est uniforme: $200 \mathrm{fcfa} / \mathrm{client}$. Toutefois, lorsqu'un taxi est pris en " course », le client paie $1000 f c f a$. En fait, ce dernier pressé ou par manque de clients accepte de déplacer le véhicule à ses seuls frais. Une journée de travail débute autour de 06 heures du matin et s'achève vers 20 heures. Selon les propos des chauffeurs «il n'y a plus de permanence comme avant». Avant 2002, les cars en provenance des villes et pays frontaliers arrivaient à Korhogo à n'importe qu'elle heure. Pour les déplacements de ces voyageurs, certains chauffeurs de taxi-voitures travaillaient jusqu'à des heures tardives. Aujourd'hui, pour des raisons de sécurité, les voyages de nuit ne sont plus admis par les autorités. Les seules permanences se font devant le CHR pour l'assistance des malades. L'organisation ci-dessus est celle des trois gares suivantes : petit Paris, Haoussabougou, CHR.

À la gare de Napié, les départs se font par ordre d'arrivée ou d'entrée en gare. Les véhicules chargent et partent de la gare les uns à la suite des autres. Les derniers départs sont à 19heures. Généralement, ils sont assurés par les chauffeurs résident Napié et/ou Dikodougou. Pour rappel, cette gare dessert uniquement la campagne. Nous avons donc à faire à des taxibrousses. 


\section{L'impact des taxi-motos sur la survie des taxi-voitures à Korhogo}

À ce stade de notre réflexion, nous analysons les différentes mutations intervenues dans la vie des chauffeurs et propriétaires de taxivilles depuis l'irruption de la moto comme mode de transport collectif à Korhogo. Dans cette optique, nous nous appuierons sur les indicateurs suivants : les gains journaliers, clientèle, le nombre de chauffeurs/véhicule, l'évolution du parc automobile, etc.

Avant la crise, le bénéfice net journalier d'un chauffeur de taxi-ville oscillait entre 10000 et 13 000fcfa. Cette somme était versée au patronpropriétaire. Quant à la rémunération du chauffeur, elle était fixée selon deux modalités : soit il a un salaire mensuel, soit il n’en a pas. Dans le premier cas, son salaire mensuel est de 30 000fcfa. Pour cela, ce dernier verse 10 000fcfa/jour. Dans le second cas, le chauffeur ne perçoit pas de salaire. Il verse donc à son patron-propriétaire 13 000fcfa/jour. En retour, les entrées des samedis et dimanches lui reviennent de droit. Aujourd'hui, le bénéfice net d'un chauffeur de taxi-ville oscille entre 5000 et $7000 \mathrm{fcfa} /$ jour. Cependant, le salaire (de $30000 \mathrm{fcfa} / \mathrm{mois}$ ) n’a pas changé. Les charges (dépenses) de fonctionnement ont connu une légère variation. En effet, on est passé de 20-15 litres/journée (avant la crise) à 10 litres/journée (aujourd’hui). Aussi, toutes les deux semaines, la vidange du véhicule doit être faite. Il en est de même pour le filtre à huile et le filtre à gasoil. Selon les chauffeurs, la vidange est fonction du kilométrage. Le phénomène taxi-moto a également entrainé des mutations sur le parc auto et le nombre de travailleur sur un taxivoiture. En effet, avant la crise la ville de Korhogo comptait environ 500 taxi-voitures. Au dernier recensement (août 2015), le syndicat a dénombré exactement 86 véhicules encore fonctionnels dans la ville de Korhogo. Par gare, on peut avoir une telle répartition: gare petit Paris (25), gare Haoussabougou (11), gare CHR (30) et la gare de Napié (30). Avant la crise, les gares de Haoussabougou et petit Paris étaient les principaux points de stationnement de la ville. Dans le contexte actuel, ils sont en train de perdre leur suprématie ; surtout Haoussabougou. À cela, s’ajoute le fait que sur un véhicule on ne trouve qu'un seul chauffeur. Ce qui n’était pas le cas auparavant. Parmi ces nombreux chauffeurs au chômage, quelques uns ont été récupérés par les grandes compagnies (EKDS, COIC, URECOS-CI) de coton que compte la ville. Ces derniers à leur moment de loisiveté se retrouvent à la gare de petit Paris se remémorant «l'ancienne époque où deux chauffeurs pouvaient facilement s'en sortir quoique travaillant sur la même voiture ». Dans une telle situation, un chauffeur était le titulaire et le second le contractuel. Ils se partageaient la journée : le jour pour l'un et la nuit pour l'autre. Toutefois, il convient de préciser que l'avènement de la moto dans le transport collectif à Korhogo n'est pas l'unique facteur qui impacte la réduction du parc auto des taxi-voitures. Ce facteur est d'ordre 
politique. En fait, dès le déclenchement de la guerre le 19 septembre 2002, l'activité des taxi-villes fut interrompue par les autorités militaires rebelles. De plus, ils ont arraché plusieurs véhicules des mains de leurs propriétaires pour les revendre en pièces détachées (nos enquêtes, 2015). Raison pour laquelle, depuis la reprise le parc n'a pas encore atteint son niveau initial. Pour les chauffeurs interrogés, "...depuis le commencement, ils sont handicapés ».

Par ailleurs, les biens et personnes transportés n’ont pas changé. Cependant, les taxi-villes perdent du terrain devant la concurrence des taximotos et tricycles.

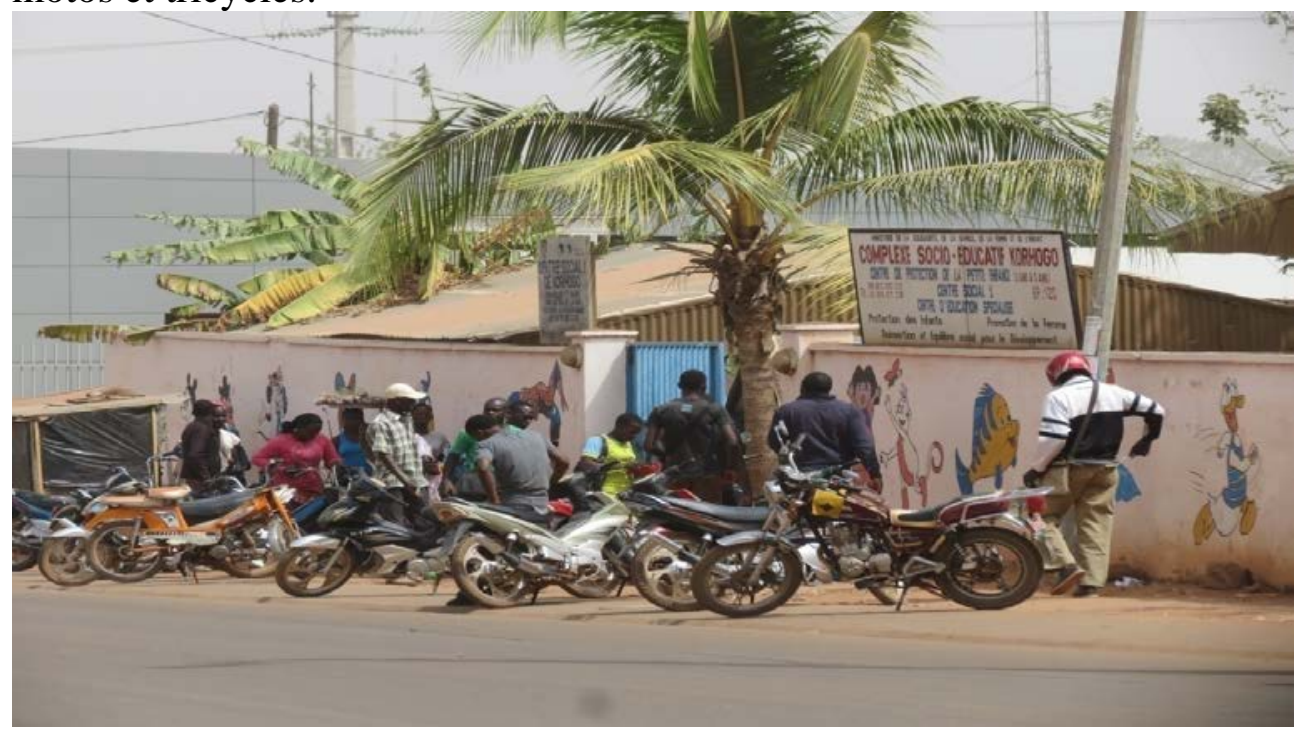

Cliché : DINDJI Roger, 2015

Figure 4 : Motos taxis et motos individuels en attente d'enfants à leur pause de $11 \mathrm{~h} 30$ devant un centre pré scolaire

Comme clientèle, les taxi-voitures ont toujours à faire aux commerçants (les femmes surtout), aux fonctionnaires, aux élèves et étudiants. Selon nos enquêtes, les femmes représentent la clientèle fidèle. Il s'agit des commerçantes des marchés d'une part et; celles qui font le ménage pour leur époux d'autre part. C'est ce qui explique la localisation des deux principales gares de taxi-voitures. En effet, elles encadrent bien le grand marché. Cette situation est bien perceptible sur la figure 3 avec la gare de petit Paris au Nord du grand marché et celle de Haoussabougou au Sud. Par ailleurs, la (figure 4) ci-dessus nous montre que les taxi-voitures sont en réel perte de «terrain ». En fait, avant 2002, les chauffeurs de taxi-voitures passaient des contrats avec des parents d'élèves pour le transport de leurs enfants. Ces derniers recevaient une rémunération mensuelle. Aussi, aux heures de descentes les chauffeurs se postaient devant les écoles pour le 
ramassage des écoliers. Aujourd'hui, tel n'est plus le cas. En effet, ils ont perdu ce marché au profit des taxi-motos.

Les taxi-villes connaissent quelques périodes de flux: jours de marchés, les prises en " course ». Il y a aussi la saison sèche. En fait, c’est la période propice pour l’organisation de funérailles chez le Senoufo (les autochtones de Korhogo). Les chauffeurs se font plus d'argent, puisqu'il y a une quantité importante de personnes à transporter. Cependant, dans le transport des élèves, fonctionnaires et bagages les taxi-villes ont perdu du terrain au détriment des motos et tricycles. Aujourd'hui, les parents d'élèves, les fonctionnaires passent plus de contrats avec les motos. Ce qui n’était pas le cas avant l'avènement de ce phénomène dans la ville.

\section{Conclusion}

De cet article, nous retenons d'abord que la consolidation du phénomène moto taxi en Côte d’Ivoire en général et à Korhogo en particulier repose sur la conjugaison de plusieurs facteurs. Les populations korhogolaises ont l'habitude de la moto. Or, dès le déclenchement de la crise politique et militaire le 19 septembre 2002, les taxi-villes ou taxi-voitures jusque là seule offre de la mobilité de masse en ville fut interdits. Profitant de ce vide la moto a commencé à rendre service pour finalement s’imposé comme mode de transport de masse. Cependant, cette situation impacte considérablement l'organisation fonctionnelle d'une part et ; menace même la survie des taxi-villes d'autre part. Malgré la création de gares pour la desserte de la ville, sur plusieurs indicateurs la concurrence balance en la faveur des taxi-motos. En effet, la recette journalière des chauffeurs de taxivilles a été divisée de moitié. Le nombre des taxi-villes est passé de 480 avant la guerre à 86 à ce jour. Se sentant lésé au profit des taxi-motos par les autorités locales, les chauffeurs de taxi-voitures ne paient plus de taxes à la mairie et aux services des impôts d'Etat. En clair, la pérennisation du taxiville semble fragile si les "gestionnaires de villes » ne prennent pas de normes garantissant plus d'équité dans le fonctionnement de ces deux modes de transports artisanaux.

\section{References:}

1. AKA KOUADIO A., (2006). Les taxis communaux ou woro woro à Abidjan-Cocody : caractéristiques, organisation et fonctionnement, In Revue de Géographie Tropicale et d'Environnement, n6, pp. 45-61.

2. ALOKO-N'GUESSAN J., (1989). Essai d'analyse des facteurs d'émergence des taxis-ville ou wôrôs-wôrôs à Abidjan, In Revue tunisienne de Géographie, $\mathrm{n}^{\circ}$ 29, pp. 41-64.

3. ASSOGBA GUEZERE, (2012). Les taxis brousses togolais: une réadaptation des taxis collectifs imposée par la concurrence des taxis- 
motos dans les villes secondaires, In Conférence CODATU XV: ''Le rôle de la modalité urbaine pour (re)modéler les villes", Addis Abeba (Ethiopie), $18 \mathrm{p}$.

4. BUGNICOURT J., (1981). Transports “'informels" ou 'transitionnels"' dans les villes du tiers-monde : les remplacer ou les promouvoir ?, In Bugnicourt J. et al.-transports en sursis ? Quelles politiques des 'petits transports"' urbains dans le tiers-monde?, Dakar/Londres, Environnement africain, pp.103-120.

5. BRUCE T., (1998). Des services de transport adaptés aux besoins d'un monde en mutations : la clé de la prospérité socio économique, Le Courrier, $\mathrm{n}^{\circ} 169$, pp. 30-34.

6. BROU E.KOFFI, (2008). Le transport de personne et la structuration de l'espace urbain de Daloa, Le Journal des Sciences Sociales, $\mathrm{n}^{\circ} 5$, pp. 127-142.

7. DINDJI ROGER, (2014). Décentralisation et développement local : cas de la commune de Cocody, thèse unique de doctorat de Géographie non publiée, Université de Cocody-Abidjan, IGT, Côte d'Ivoire, $282 \mathrm{p}$.

8. GODARD X. et TEURNIER P., (1992). Les transports urbains en Afrique à l'heure de

9. l'ajustement, Paris, Editions Karthala-INRETS.

10. KASSI I. DJODJO, (2010). Rôles des transports populaires dans le processus d'urbanisations à Abidjan, Les Cahiers d'Outre-mer, n'251, pp. 391-402.

11. LOUISE DIAZ O. et Al., (2007). La diffusion des taxis-motos dans l'Afrique urbaine au Sud du Sahara, In XLIIIème colloque de l'ARSDLF, Grenoble-Chambéry, 17 p.

12. PIERRE KAMDEM et Al., (2007). L'intégration des motos-taxis dans le transport public au Cameroun ou l'informel à la remorque de l'Etat : une solution d'avenir au problème de mobilité et de l'emploi en Afrique subsaharienne, In Colloque international de Lisbonne, pp. $1-17$.

13. TUBLU KOMI F., (2010). Le taxi-moto: un nouveau mode de transport dans les mobilités urbaine au Togo pour quelle qualité de vie?, In Conférence CODATU XIV Buenos Aires (Argentine): transport durable et qualité de vie en ville, pp. 1-12.

14. TUO PEGA, (2013). Approche géographique de la méningite et du paludisme dans le Nord ivoirien : le cas de Korhogo, thèse unique de doctorat de Géographie non publiée, Université de Cocody-Abidjan, IGT, Côte d'Ivoire, 324 p.

15. ZOUHOULA-BI M.R., (2010). Les fondements de la légitimité de l'emprise des corporations syndicales sur la frange informelle des 
transports urbains à Abidjan, In Conférence CODATU XIV Buenos Aires (Argentine) : transport durable et qualité de vie en ville, pp. 116. 